\title{
Strange New Worlds: Comparative Planetology of Exoplanets and the Solar System
}

\section{Primary author:}

Ravi Kopparapu ${ }^{1}$

(ravikumar.kopparapu@nasa.gov)

301-286-1548

\section{Co-authors}

Giada Arney ${ }^{1}$, Shawn Domagal-Goldman', Edward Schwieterman ${ }^{3}$, Hilairy E. Hartnett', Nancy Y. Kiang, Evan L. Sneed ${ }^{16}$, Christopher T. Reinhard ${ }^{22}$, Chuanfei Dong ${ }^{23}$, Eric Hébrard ${ }^{24}$, Ariel Anbar ${ }^{31}$, Nuno C Santos ${ }^{32}$, Neal Turner ${ }^{14}$

\section{Co-signees}

Vladimir Airapetian ${ }^{1,2}$, Stephen R. Kane', Jeremy J. Drake ${ }^{4}$, Betul Kacar', Aki Roberge ${ }^{1}$, Thomas J. Fauchez ${ }^{1,6}$, Melissa G. Trainer ${ }^{1}$, Natalie Hinkel ${ }^{10}$, Joe P. Renaud ${ }^{1{ }^{16}}$, Knicole Colón ${ }^{1}$, Carey Lisse ${ }^{7}$, John Ahlers ${ }^{1,6}$, Dawn M. Gelino', Jonathan Fortney ${ }^{11}$, Jacob Haqq-Misra ${ }^{12}$, Seth Redfield ${ }^{13}$, Nader Haghighipour ${ }^{15}$, P. Wilson Cauley ${ }^{17}$, Paul Kalas ${ }^{19}$, Daria Pidhorodetska ${ }^{13,18}$, William Cochran ${ }^{20}$, Jacob Lustig-Yaeger ${ }^{21}$, Nathan Mayne ${ }^{24}$, William B. Moore ${ }^{25}$, Wesley D. Swingley ${ }^{26}$, William C. Danchi ${ }^{1}$, Siegfried Eggl ${ }^{21}$,David Grinspoon ${ }^{27}$, Scott D. Guzewich", Michael J. Way ${ }^{8}$, Rajdeep Dasgupta ${ }^{34}$, Robin Wordsworth $^{28}$, Kazumi Ozaki ${ }^{29}$, Chris Stark ${ }^{30}$, Courtney Dressing ${ }^{19}$, Avi Mandell ${ }^{1}$, Patrick Young ${ }^{31}$, Yuni Lee $^{1,1,3,33}$, Jessica Noviello ${ }^{31}$, Sang-Heon Shim ${ }^{7}$, Hannah Jang-Condell ${ }^{35}$, Kathleen J. McIntyre ${ }^{36}$, Niki Parenteau ${ }^{37}$, Karalee K. Brugman ${ }^{7,38}$, Peter Gao ${ }^{11,19}$, Kaveh Pahlevan ${ }^{39}$, J. Serena Kim ${ }^{40}$, Kevin Stevenson ${ }^{41}$, Thomas G. Beatty ${ }^{5}$, Giannina Guzman ${ }^{1,2,42}$, Corrine Rojas ${ }^{7}$, Shirin G. Zaidi ${ }^{16}$, Gudmundur Stefansson ${ }^{23}$, Monica Vidaurri ${ }^{1,43}$, David R. Ciardi ${ }^{9}$, Wladimir Lyra ${ }^{44}$, Kyle Batra ${ }^{16}$, Timothy Lyons ${ }^{3}$, Victoria S. Meadows ${ }^{21}$, Julian M. Chesnutt ${ }^{46}$, Jaime A. Cordova Jr. ${ }^{44}$, Sarah Rugheimer ${ }^{47}$

NASA Goddard Space Flight Center, Greenbelt, MD, USA, 2American University, DC ${ }^{3}$ UC Riverside, "Center for Astrophysics | Harvard \& Smithsonian, sUniversity of Arizona, Universities Space Research Association (USRA), 'Arizona State University, Tempe, AZ, ${ }^{8} N A S A$ Goddard Institute for Space Studies, ${ }^{\circ}$ NASA Exoplanet Science Institute (NExScl), IPAC/Caltech, ${ }^{10}$ Southwest Research Institute, San Antonio, TX, ${ }_{11}$ University of California, Santa Cruz, ${ }^{2 B} B l u e$ Marble Space Institute of Science, ${ }^{13}$ Wesleyan University, ${ }^{14} \mathrm{JL}$, California Institute of Technology, ${ }^{15}$ Institute for Astronomy, University of Hawaii-Manoa, ${ }^{10}$ Pennsylvania State University, University Park, PA, ${ }^{1} \mathrm{~L}$ LSP, University of Colorado Boulder, ${ }^{18}$ The Center for Research and Exploration in Space Science and Technology II (CRESST II), ${ }^{19}$ University of California, Berkeley, ${ }^{20}$ University of Texas at Austin, ${ }^{2}$ University of Washington, ${ }^{22}$ Georgia Institute of Technology, ${ }^{23}$ Princeton University, ${ }_{24}$ University of Exeter, ${ }^{25} \mathrm{Hampton}$ University, ${ }^{26}$ Northern Illinois University, ${ }^{27}$ Planetary Science Institute, ${ }^{28} \mathrm{Harvard}$ University, ${ }^{29}$ Toho University, ${ }^{30}$ Space Telescope Science Institute, ${ }^{31}$ Arizona State University, ${ }^{32}$ Instituto de Astrofísica e Ciências do Espaço and Departamento de Física e Astronomia, Univ. Porto, Portugal, ${ }_{33}$ University of Maryland, Baltimore County, Baltimore, MD, ${ }^{4}$ Department of Earth, Environmental, and Planetary Sciences, Rice University, Houston, TX, 
${ }^{35}$ University of Wyoming, Laramie, WY, ${ }^{36}$ University of Central Florida, Orlando, FL, ${ }^{37}$ NASA Ames Research Center, ${ }^{38}$ Carnegie Institution for Science, Washington, D.C., ${ }^{39}$ SETI Institute, ${ }^{40}$ Steward Observatory, University of Arizona, ${ }^{41}$ Johns Hopkins APL, Laurel, MD, USA, ${ }^{42}$ University of Maryland, College Park, MD, ${ }^{43}$ Howard University, ${ }^{44}$ New Mexico State University

\begin{abstract}
This whitepaper discusses the striking diversity of exoplanets that could be detected by future observations and highlights the potential for comparative planetology to be performed with Solar System planets in the upcoming era of large, space-based flagship missions. The primary science focus will be on characterizing Earth-like worlds around Sun-like stars. However, we will simultaneously characterize companion planets. This characterization effort will not only provide a contextual picture with regard to our Solar System, but also presents a unique opportunity to observe correlations between atmospheric composition, planetary size, orbital distance, etc. Whether they orbit our Sun or another star, planets are planets. The field of 'planetary science' should embrace a unified approach to the study of planets in which questions and insights from exoplanets and from objects in our Solar System flow seamlessly. To that end, we recommend (1) continued support of community-wide cross-disciplinary efforts that promote communication and collaborative venues for scientists in both the fields and (2) dedicated effort to apply the modeling techniques ("lessons-learned") of the Solar system objects to exoplanet parameter regime, to predict observables.
\end{abstract}

Within the past two decades, the number of confirmed exoplanets has increased a thousandfold, with thousands more waiting to be confirmed (e.g. Mullally et al. 2017, NASA Exoplanet Archive). In the past few years alone, several habitable zone (HZ) planets around the nearest $\mathrm{M}$ dwarfs have been discovered (Anglada-Escudé et al. 2016; Gillon et al. 2017, Dittman et al. 2017). Detecting Earth-like planets around solar-type stars is one of the main science drivers of future space photometric transit missions like PLAnetary Transits and Oscillations of stars PLATO (Rauer et al. 2014). Masses for these planets will come from precise follow-up with ground based high resolution spectrographs like ESPRESSO (Pepe et al. 2014), EXPRES (Jurgenson et al. 2018) and NEID (Schwab et al. 2016).

While the efforts to identify habitable exoplanets are receiving increasing attention, a wide variety of planetary objects have also been discovered which have no analog in our Solar System, such as super-Earths, sub-Neptunes and hot Jupiters, many of them orbiting their host stars closer than Solar system planets to our Sun. Future large space-based telescopes will have the capabilities to detect and characterize a multitude of these planets, along with Earth-like worlds. Studies of these worlds will help to place the Solar System into a larger context and help us to understand how common - or uncommon - the planets and architecture of our Solar System are on a wider scale. Understanding this large diversity of planets calls for interdisciplinary efforts to develop a new field of comparative planetology. Organizationally and culturally, "exoplanets" are the domain of astronomy, while solar system planets are the domain of "planetary scientists". While this is sociologically and historically understandable, it is increasingly an obstacle to scientific progress. 
We summarize below the potential parameter space that may be sampled with upcoming missions and the roles that a variety of scientific disciplines can play to develop generalized principles that further our understanding of planetary systems and help us search for the life that may exist in them.

With the Transiting Exoplanet Survey Satellite (TESS) and Gaia telescopes, the next decade will see a vast increase in the number and diversity of exoplanet discoveries. The James Webb Space Telescope (JWST) will help characterize atmospheres of some of these planets and the Nancy Grace Roman Space Telescope (formerly WFIRST) will image some while developing starlight suppression technologies and exozodiacal light subtraction algorithms for future large-aperture space-based direct imaging missions. Soon after, ground-based facilities like the Extremely Large Telescope (ELT) and Giant Magellan Telescope (GMT) (Rodler \& López-Morales 2014), and missions like the Large UV / Optical / Infrared Surveyor (LUVOIR, LUVOIR Team et al. 2020), Habitable Exoplanet Observatory (HabEX, Gaudi et al. 2020) and Origins Space Telescope (Meixner et al. 2019) concepts will take the lead in characterizing atmospheres of $\mathrm{HZ}$ planets around GKM stars via transit spectroscopy, direct imaging and secondary eclipse/thermal phase curves. These missions, combined with the study of "model exoplanets" within our Solar System, either by missions or remote observations, are a crucial first step in establishing the new field of comparative planetology.

In future searches for exo-Earth candidates around nearby Sun-like stars, planet search yield simulations suggest that we may detect several bright planets (Beckwith 2008). According to Stark et al. (2015), an 8-meter telescope observing 500 stars would be able to detect 20 exoEarth candidates (see Fig. 1 in Stark et al. 2015), although this is strongly dependent on the value of $\eta_{\text {Earth, }}$ the fraction of stars that have at least one terrestrial mass/size planet in the habitable zone. If we assume that, on average, every star has a planet of any size (Cassan et al. 2012; Suzuki et al. 2016), then there are $~ 500$ exoplanets of all sizes that could be observed with this type of facility, providing an astonishing sample of planetary diversity. Beyond the 20 exo-Earth candidates, most exoplanets will fall into the "non-Earth" classification. However, thus far there is no universally accepted classification system for distinguishing among them. This provides a motivation to devise a schema based on planetary size and corresponding comparative atmospheric characteristics to differentiate features between separate classes of non-Earth-like planets.

Classifying planets of different sizes based on the transition/condensation of different atmospheric species (Sudarsky et al. 2003; Burrows 2005) at different stellar fluxes and stellar properties (e.g. mass, metallicity, evolutionary state) provides a physical motivation in estimating exoplanet mission yields distinct from exo-Earth candidate yields (Fig. 1). Mission yields are defined as the numbers of exoplanets in specific classes detected and spectroscopically characterized by a direct imaging mission. Additionally, the interior structure and composition of the planet affects the atmospheric temperature profile (Wordsworth et al. 2018). This, in turn, will impact the condensation of minerals, and therefore structural boundaries and planetary composition (Hinkel \& Unterborn, 2018). A classification scheme is shown in Fig. 1. Radius estimates are from Zahnle \& Catling (2017), Fulton et al. (2017) and Chen \& Kipping (2017). 


Using multiple
observation techniques
will provide the best
information on
exoplanet atmospheres.
Optical color-color
photometry may provide
a zeroth order
categorization of planet
types. However, some
planetary atmospheres
will be more difficult to
characterize than others.
For example, in our Solar
System, clouds and
hazes are ubiquitous,
and the transit
observations that will be used for next-generation exoplanet observation platforms like JWST can only sense the

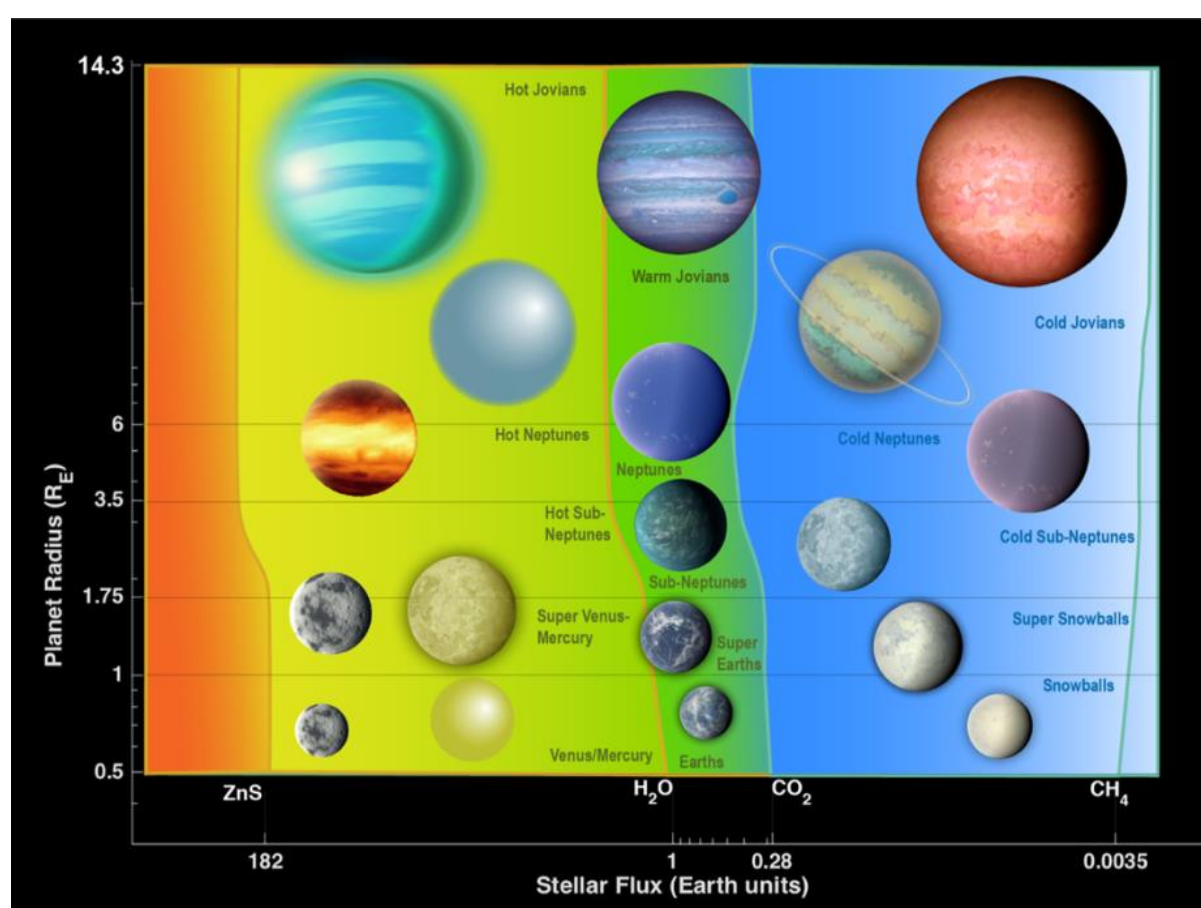

Fig. 1: The boundaries of the boxes represent where different chemical species are condensing in the atmosphere of a planet at a stellar flux, according to equilibrium chemistry calculations (Kopparapu et al. 2018).

uppermost reaches of a cloudy/hazy atmosphere. Secondary eclipse and phase curve measurements, which sense directly emitted thermal light, are less vulnerable to truncation by clouds and hazes and may allow access to molecules closer to the surface of the planet. Direct imaging in reflected light as a function of orbital phase at UV-optical-NIR wavelengths will provide access to broadband UV absorption features, as well as other visible and NIR absorbers, potentially yielding evidence of surface composition and liquid water at the surface. In the end, the most complete understanding of an exoplanet will combine multiple observation techniques across the widest possible wavelength range.

There is already a need for a coordinated interdisciplinary effort to interpret spectroscopic observations of exoplanets, and this effort must naturally involve Solar System scientists (Charnay et al. 2015, Hammond \& Pierrehumbert 2017, Kane et al. 2020 whitepaper). Currently, comparative planetology studies are limited (by both sensitivity and spectral resolution) to some bulk properties of a collection of mostly hot Jupiter-type planets (e.g., Barstow et al. 2016). The JWST mission will have the capacity to vastly expand exoplanetology to various other planet categories including hot, warm, and cold Neptunes and sub-Neptunes along with a limited number of terrestrial planets, including super-Earths, Earths, and Venus analogs (Cowan et al. 2015; Greene et al. 2016). Combined with statistical information from the Nancy Grace Roman Space Telescope, this comprehensive dataset will allow unprecedented insight into compositional trends and will impose meaningful new constraints on planet formation models 
(e.g., Espinoza et al. 2017; Venturini et al. 2016). The European Atmospheric Remote-sensing Infrared Exoplanet Large-survey (ARIEL) mission (Tinetti et al. 2016) will allow us to extract the chemical fingerprints of gases and condensates in the atmospheres of a large number of hot-towarm planets and statistically analyze these planets' elemental compositions. Near- and mid-IR space interferometry missions (Quanz et al. 2018) to directly image exoplanets around nearby Sun-like stars will provide information about molecular features using spectral bands from molecules such as carbon dioxide, water vapor, nitrous oxide, methane, hydroxyl and nitric oxide (Airapetian et al. 2017; 2020). Future large-aperture, direct-imaging missions such as LUVOIR and HabEx will extend comparative exoplanet studies by observing systems of sibling planets with diverse compositions. It is noteworthy that the study of secondary atmospheric escape and the evolution of terrestrial exoplanets is a rapidly growing field of research (Dong et al. 2018, 2020), and LUVOIR and HabEx will further facilitate this topic with future observations. Here especially, interdisciplinary collaborations will be required for the interpretation of the data.

In some circumstances, a planet's internal heat flux will significantly modify its surface temperature relative to the stellar flux. For very close-in worlds with modest-to-high eccentricity maintained by nearby (resonant or non-resonant) perturbers, tidal heating may contribute millions of times the heat output of the modern Earth but only a 1-5 $\mathrm{K}$ variation in surface temperature (Henning et al. 2009). Such issues become more complex for tidally locked dayside/nightside thermal dichotomies. Conversely, for some icy exomoons, where insolation is weak, internal heat flux variations will matter more, but may still fit the scheme shown in Fig. 1 using a combined internal-solar heat flux axis.

The histograms in Fig. 2 visualize comprehensive exoplanet yield by size and temperature of the habitable planet candidate survey, along with the several other classes of exoplanets, based on 4-m and 16-m diameter telescopes. The $y$-axis in Fig. 2 is the expected total number of exoplanets observed (i.e., yield; also given by the numbers above the bars). For the large architectures (16$\mathrm{m}$ telescopes, analogous to LUVOIR-A), one can easily see the diversity in exoplanet yields and begin to further characterize different classes of planets. We note that in general, larger apertures are less sensitive to changes in other parameters (such as contrast ratio) than smaller apertures.

With a 4-m class mission (analogous to HabEx Coronagraph), observations designed to maximize the yield of potential Earths will also yield detections and characterization of all of the planet types discussed here, with the exception of close-in planets. These planets are not observable by a 4-m class mission because of the tight inner working angle.

The 16-m class telescopes would bring the ability to not only characterize planets, but to test the occurrence of features within each planet type. They would observe dozens of each type, providing samples large enough to enable the study of each type as a population. Furthermore, a large direct detection mission with UV-to-NIR spectral coverage characterizes the host star's high energy irradiance that regulates the atmospheric composition and stability on all planets (Harman et al. 2015; Koskinen et al. 2013), while also characterizing the exozodiacal dust that 
reveals the alien planetary system's analogs to the solar system's asteroids and comets (Bonsor et al. 2012).
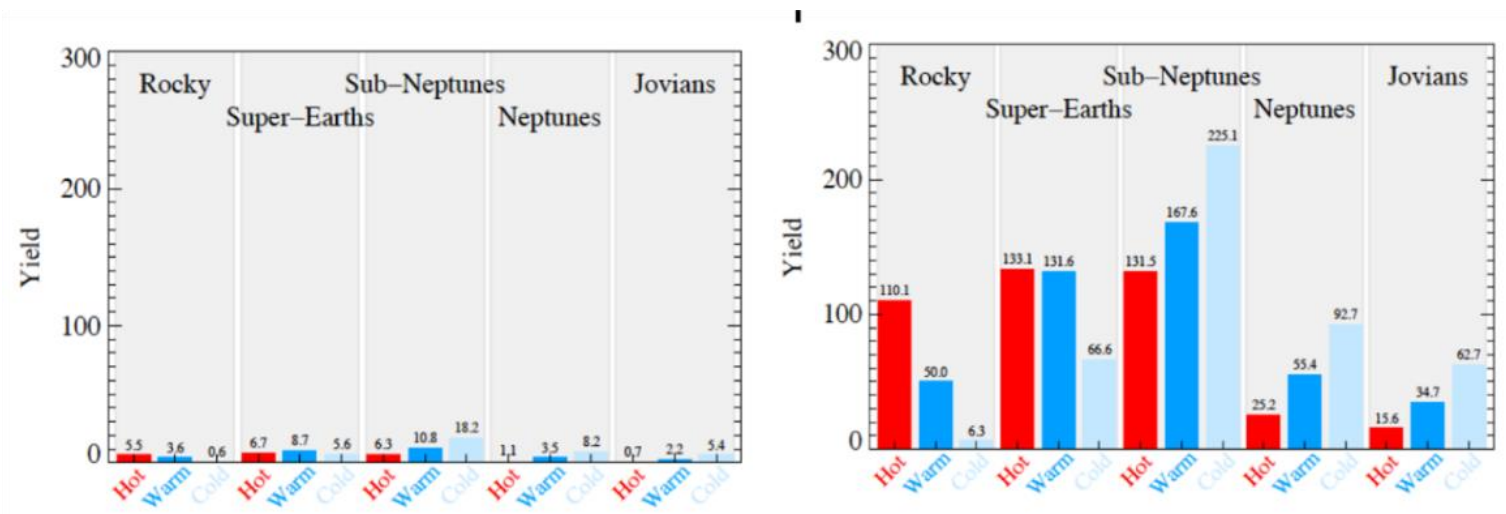

Fig. 2: Expected number of exoplanets observed for hot (red), warm (blue) and cold (ice-blue) incident stellar fluxes shown in Fig. 1. Calculations assume a coronagraph paired with a telescope of diameter 4-m (left) and 16-m (right).

Approaches must be developed for the identification and classification of exoplanet diversity, which will require expertise in theory and experiments in the planetary science, climate science, astrophysics, chemistry, evolutionary biochemistry, and stellar/heliophysics communities. Understanding the atmospheric chemical composition and condensation based on the global temperature profile of an exoplanet requires a coordinated interdisciplinary effort. To that end, we recommend continued support for a community-wide effort to foster communication and collaborative venues for cross disciplinary scientists is needed. One such venue was the "Exoplanets in Our Backyard" conference in February at Houston, where both exoplanet and Solar System scientists came together for a focused and in-depth discussion of cutting-edge research in their respective fields. This was a hugely popular, and highly successful meeting, that spurred collaborations between the communities. In particular, the meeting identified a key avenue where a collaboration between solar system and exoplanet communities would be highly impactful. Most of the exoplanets discovered to-date are in close-in orbits, receiving high stellar radiation compared to Solar system planets. Furthermore, some classes of objects either are not found in the Solar system (super-Earths and sub-Neptunes) or probably scaled-up versions of the extremely-water-rich giant-planet moons. An interdisciplinary, dedicated effort to apply the modeling techniques ("lessons-learned") of the Solar system objects to exoplanet parameter regime, and predicting observables is recommended. The vast quantity of exoplanets that will be available for atmospheric characterization in the near future provides us with a golden opportunity to perform comparative exoplanetology. Establishing this new field of comparative planetology ultimately paves the way to new understandings of habitability, and perhaps one day, the discovery of life on other planets.

\section{Reference}

Airapetian, V. S., Barnes, R., Cohen, O. et al. 2020, Int. J. of Astrobio., 19,136 
Reference

Airapetian, V. S., Barnes, R., Cohen, O. et al. 2020, Int. J. of Astrobio., 19,136

Airapetian, V. S., Jackman, C., Mlynzcak, M. et al. 2017, Nature Sci. Rep. 7

Anglada-Escudé, G., Amado, P. J., Barnes, J. et al. 2016, Nature, 536, 7617, 437

Barstow, J. K., Aigrain, S., Irwin, P. G. J., \& Sing, D. K. 2016, ApJ 834, 50

Bean, J. L., Abbot, D. S., \& Kempton, E. M. -R. 2017. ApJL, 841, L24

Beckwith, S. V. W. 2008. ApJ, 684, 1404

Bonsor, A., Augereau, J.-C., Thébault, P. 2012, A\&A 548, A104

Burrows, A. 2005. Nature, 433, 261

Charnay, B., Meadows, V. S., Misra, A. et al. 2015. ApJL, 813, L1

Chen, J., \& Kipping, D. 2016. ApJ, 834, 17

Cowan, N. B., Greene, T., Angerhausen, D., et al. 2015, PASP, 127, 311

Crow, C.A., McFadden, L. A. , Robinson, T., et al. 2011, ApJ 729, 130

Dong, C., Jin, M., Lingam, M. et al. 2018, PNAS, 115, 260

Dong, C., Jin, M., Lingam, M. 2020, ApJL, 896, L24

Dittman, J. A., Irwin, J. M., Charbonneau, D. et al. 2017. Nature, 544, 333

Espinoza, N., Fortney, J. J., Miguel, Y., et al. 2017, ApJL, 838, L9

Forget, F., \& Leconte, J. 2014, Phil. Trans. R. Soc. A 372: 20130084

Fulton, B., J., Petigura, E. A., Howard, A. W., et al. 2017, AJ, 154, 3

Gaudi et al. 2020, http://arxiv.org/abs/2001.06683

Gillon, M., Triaud, A. H. M. J., Demory, B. O. et al. 2017. Nature, 542, 456

Greene, T. P., Line, M. R., Montero, C., et al. 2016, ApJ, 817, 17

Hammond, M. \& Pierrehumbert, R. T. 2017, ApJ, 849, 152

Harman, C. E., Schwieterman, E. W., Schottelkotte, J. C. 2015, ApJ, 812, 137

Henning, W. G., O'Connell, R. J., Sasselov, D. D., 2009 ApJ, 707, 1000

Hinkel, N.R., \& Unterborn, C. T., 2018, ApJ, 853, 83

Jurgenson, C., Fischer, D., McCracken, T, et al. 2016, Proceedings of the SPIE, Volume 9908

Kane, S. R., Kopparapu, R. K., Domagal-Goldman, S. 2014. ApJ, 794, L5

Kane, S. R. et al. 2020, Planetary Science Decadal Survey White paper

Kopparapu, R. K., Hebrard, E., Belikov, R., et al. 2018, ApJ, arXiv:1802.09602.

Koskinen, T. T., Harris, M. J., Yelle, R. V., Lavvas, P. 2013. Icarus, 226, 2

Krissansen-Totton J., Schwieterman E. W., Charnay B. et al 2016 ApJ 8171

LUVOIR Team et al. (2019), http://arxiv.org/abs/1912.06219

Meixner, M. et. al. (2019), arXiv http://arxiv.org/abs/1912.06213

Pepe et al. 2014, Astronomische Nachrichten, Vol.335, Issue 1, p.8

Pierrehumbert, R. T., \& Ding, F. 2016. The Royal Society 472, 20160107

Quanz, S. P. et al. (2018) Optical and Infrared Interferometry and Imaging VI. SPIE, p. 37. doi:

$10.1117 / 12.2312051$

Rauer et al. 2014, Experimental Astronomy, Volume 38, Issue 1-2, pp. 249-330

Rodler, F., \& López-Morales, M. 2014. ApJ, 781, 54

Schaefer, L., Wordsworth, R., Berta-Thompson, Z., Sasselov, D. 2016. ApJ, 829, 63

Stark, C. C. et al. 2015. ApJ, 808, 149

Sudarsky, D., Burrows, A., Hubeny, I. 2003. ApJ, 588, 1121

Suzuki, D., Bennett, D. P., Sumi, T. 2016. ApJ, 833, 145 
Tinetti, G. et al. (2016) SPIE, p. 99041X. doi: 10.1117/12.2232370

Venturini, J., Alibert, Y., \& Benz, W. 2016, A\&A, 596, A90

Wordsworth, R., Schaefer, L., Fischer, R. submitted to ApJ, arxiv:1710.00345

Zahnle, K. J., \& Catling, D. C. 2017. ApJ, 843, 122 